\title{
Parental mosaicism is a pitfall in preimplantation genetic diagnosis of dominant disorders
}

\author{
Julie Steffann $^{\star, 1,2}$, Caroline Michot ${ }^{1,2}$, Roxana Borghese ${ }^{1,2}$, Marcia Baptista-Fernandes ${ }^{1,2}$, Sophie Monnot ${ }^{1,2}$, \\ Jean-Paul Bonnefont ${ }^{1,2}$ and Arnold Munnich ${ }^{1,2}$
}

PCR amplification on single cells is prone to allele drop-out (PCR failure of one allele), a cause of misdiagnosis in preimplantation genetic diagnosis (PGD). Owing to this error risk, PGD usually relies on both direct and indirect genetic analyses. When the affected partner is the sporadic case of a dominant disorder, building haplotypes require spermatozoon or polar body testing prior to PGD, but these procedures are cost and time-consuming. A couple requested PGD because the male partner suffered from a dominant Cowden syndrome (CS). He was a sporadic case, but the couple had a first unaffected child and the non-mutated paternal haplotype was tentatively deduced. The couple had a second spontaneous pregnancy and the fetus was found to carry the at-risk haplotype but not the PTEN mutation. The mutation was present in blood from the affected father, but at low level, confirming the somatic mosaicism. Ignoring the possibility of mosaicism in the CS patient would have potentially led to selection of affected embryos. This observation emphasizes the risk of PGD in families at risk to transmit autosomal-dominant disorder when the affected partner is a sporadic case.

European Journal of Human Genetics (2014) 22, 711-712; doi:10.1038/ejhg.2013.164; published online 11 September 2013

Keywords: preimplantation genetic diagnosis; dominant disorders; mosaicism; error risk

Dominant disorders represent the most frequent cause of request for molecular preimplantation genetic diagnosis (PGD, $\sim 30 \%$ of requests in France, Agence de la Biomedecine, accessible on http:// www.agence-biomedecine.fr/annexes/bilan2011/donnees/diag-prenat/ 03-preimpl/synthese.htm). The high transmission risk in these disorders (50\%) probably explains the interest of at-risk couples for PGD. However, this risk can be overestimated owing to either somatic or germline mosaicism.

Here, we report on a family who requested PGD for CS. This diagnosis was recently made in a 32-year-old male, after colonoscopy for abdominal pains, showing ganglioneuromatosis. An oligodendrogliome of the cerebellum had been removed when he was 21 years old, with a sequelar cerebellar syndrome. A melanoma was also diagnosed when he was 27 years. His sister also has had a melanoma in her $20 \mathrm{~s}$. The couple requested PGD as they did not want to take the risk of having a child affected with CS. They were referred to our center with the molecular diagnosis of heterozygous PTEN mutation in exon 7 (c.675T $>$ A, p.Tyr225*) in blood DNA from the male partner. This mutation was neither detected in blood genomic DNA from his parents, nor in his sister, suggesting that it has occurred de novo in this patient. Because the mutation was also absent in the first son of the couple, the affected and unaffected paternal haplotypes were tentatively deduced from the study of III1 (Figure 1). Because II2 was a sporadic case, single-spermatozoon study was proposed to the couple, in order to confirm the haplotype study. ${ }^{1}$ However, a second pregnancy spontaneously occurred and prenatal diagnosis was performed in our center.

While the fetus had inherited the proposed 'at-risk' paternal allele, the c.675T $>$ A mutation was absent in the fetal DNA (Figure 1). The father II2 was found to be mosaic for the PTEN mutation in blood lymphocytes. Sequencing PTEN exon 7 using two different set of primers revealed that the mutation was present in $<20 \%$ of all sequences analyzed (Figure 1).

De novo mutations have been reported in at least $10 \%$ of PTEN hamartoma tumor syndromes. Whether these cases resulted from somatic or germline mosaicism in a parent is not known.,3 The first case of proven somatic PTEN mutation mosaicism has been recently reported, ${ }^{4}$ suggesting that this event may not be very frequent. In this latter case, the patient exhibited mild CS features. ${ }^{4}$ In our case, the proband had a history highly suggestive of PTEN hamartoma tumor syndrome. Nothing suggested that the mutation could be present at a mosaic state. Mosaicism was not mentioned in the first molecular report of the PTEN test, either, preventing us from assuming this issue.

Ignoring mosaicism in the affected partner of a couple may have important implications for PGD. Building haplotypes based on the status of the first child only would have resulted in assigning the mutation to the non-transmitted chromosome, which is the non-mutant one. Ignoring the possibility of somatic mosaicism would have therefore resulted in misdiagnosis for this couple. To decrease the risk of misdiagnosis, the ESHRE guidelines specify that when no additional family members are available for analysis in cases of paternally linked disorders, single sperm should be used for haplotype construction. ${ }^{1}$ However, single sperm analysis is sometimes complicated by sperm abnormalities (oligo- or azoospermia). In these cases, or in maternally linked disorders, even if the PGD analysis relies on both direct and indirect diagnosis with microsatellite markers flanking the PTEN gene, the possibility of allele drop-out (amplification of only one of the two alleles present in one cell)

${ }^{1}$ Unité Université Paris Descartes-Sorbonne Paris Cité, Institut imagine, INSERM-U781, Paris, France; ${ }^{2}$ Service de génétique, Hôpital Necker-Enfants Malades, Assistance Publique Hôpitaux de Paris, Paris, France

${ }^{*}$ Correspondence: Dr J Steffann, Service de génétique, Hôpital Necker-Enfants Malades, Assistance Publique Hôpitaux de Paris, Paris, France. Tel: + 33144381747 ; Fax: +33 1711964 20; E-mail: julie.steffann@inserm.fr

Received 2 April 2013; revised 3 June 2013; accepted 25 June 2013; published online 11 September 2013 


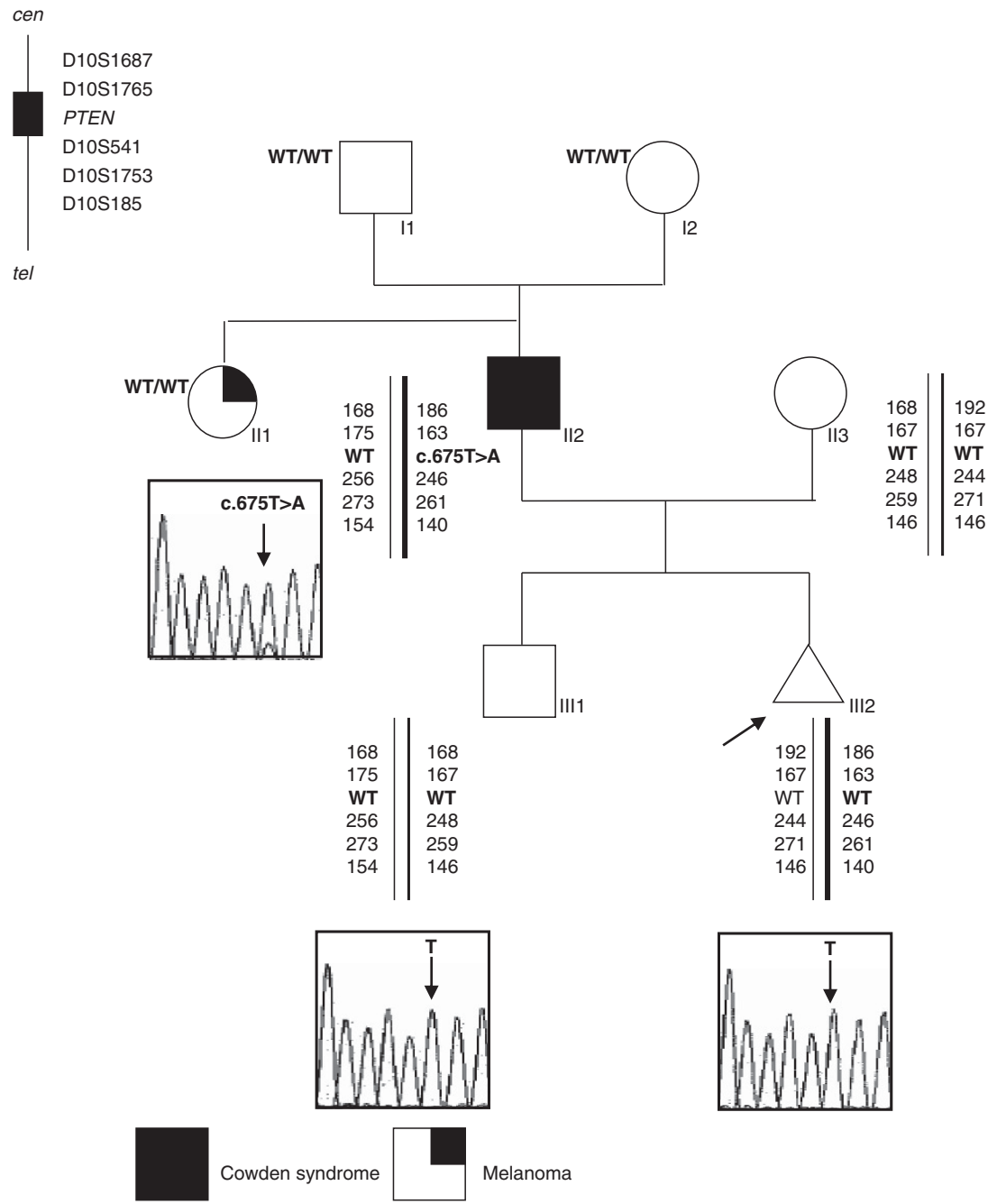

Figure 1 Pedigree of the Cowden syndrome family. Haplotypes were deduced in II2 and II3 from the study of III1. Alleles from top to bottom are ascribed to polymorphic markers from centromere to telomere. Respective locations are from UCSC genome at http://genome.ucsc.edu. WT, wild-type sequence of PTEN exon 7.

may have resulted in the transfer of mutated embryos. Keeping in mind that somatic mosaicism is a pitfall in PGD is therefore very important, particularly for conditions usually taken in charge in PGD, where the causative genes are prone to somatic mosaicism. For example, the rate of parental mosaicism averages $16 \%$ in dominant osteogenesis imperfecta families. ${ }^{5}$ Similarly, somatic mosaicism was identified in $6 \%$ of the patients with tuberous sclerosis. ${ }^{6}$ Mosaicism is also frequent in other hereditary cancer syndromes, namely neurofibromatosis 2 and bilateral vestibular schwannomas (33\%) and sporadic 'familial' adenomatous polyposis coli $(21 \%)$. $^{7}$

This observation emphasizes the risk of PGD in families at risk to transmit autosomal-dominant disorders when the affected partner is a sporadic case. This is also true when the causative gene is not known to be prone to somatic mosaicism. This pitfall can be circumvented by spermatozoon or polar body testing prior to PGD. Yet, these procedures are cost and time-consuming. This report also shows that careful laboratory analysis can detect mosaicism and emphasizes the need to report such results, as they can have major consequences for genetic counseling and management of PGD.

\section{CONFLICT OF INTEREST}

The authors declare no conflict of interest.

1 Harton GL, De Rycke M, Fiorentino $F$ et al: European Society for Human Reproduction and Embryology (ESHRE) PGD Consortium: ESHRE PGD consortium best practice guidelines for amplification-based PGD. Hum Reprod 2011; 26: 33-40.

2 Mester J, Eng C: Estimate of de novo mutation frequency in probands with PTEN hamartoma tumor syndrome. Genet Med 2012; 14: 819-822.

3 Sutphen R, Diamond TM, Minton SE, Peacocke M, Tsou HC, Root AW: Severe Lhermitte-Duclos disease with unique germline mutation of PTEN. Am J Med Genet 1999; 82: 290-293.

4 Gammon A, Jasperson K, Pilarski R, Prior T, Kuwada S: PTEN mosaicism with features of Cowden syndrome. Clin Genet 2012; e-pub ahead of print 14 December 2012.

5 Pyott SM, Pepin MG, Schwarze U, Yang K, Smith G, Byers PH: Recurrence of perinatal lethal osteogenesis imperfecta in sibships: parsing the risk between parental mosaicism for dominant mutations and autosomal recessive inheritance. Genet Med 2011; 13: 125-130.

6 Qin W, Kozlowski P, Taillon BE et al: Ultra deep sequencing detects a low rate of mosaic mutations in tuberous sclerosis complex. Hum Genet 2010; 127: 573-582.

7 Kehrer-Sawatzki H, Cooper DN: Mosaicism in sporadic neurofibromatosis type 1: variations on a theme common to other hereditary cancer syndromes? J Med Genet 2008; 45: 622-631. 